\title{
NUMERICAL SIMULATIONS OF LINEAR $S=1$ FERRO- AND ANTIFERROMAGNETS
}

\author{
G. Kamieniarz, R. Matysiak
}

Computational Physics Division, Institute of Physics, A. Mickiewicz University Umultowska 85, 61-614 Poznan, Poland

A. Caramico D'Auria, F. Esposito and U. Esposito

Dipartimento di Scienze Fisiche, Universita di Napoli, Pizzale Tecchio, Napoli, Italy

The finite-temperature static properties of the spin $S=1$ antiferromagnetic Heisenberg chains are extensively simulated using the quantum transfer matrix method. The zero-field susceptibility and specific heat as well as the field-dependent magnetization data are evaluated to select the microscopic parameters of a number of real quasi-one-dimensional compounds and to verify some theoretical approaches.

PACS numbers: $64.60 .-\mathrm{i}, 75.10 . \mathrm{Hk}, 75.40 . \mathrm{Mg}$

Antiferromagnetic quantum spin chains have attracted a lot of theoretical and experimental interest since Haldane conjecture about the difference between the ground state of integer and half-integer spins. The representative physical realization of the one-dimensional Heisenberg antiferromagnets (1D HAF) is $\mathrm{Ni}^{2+}(S=1)$ compound $\mathrm{Ni}\left(\mathrm{C}_{2} \mathrm{II}_{8} \mathrm{~N}_{2}\right)_{2} \mathrm{NO}_{2} \mathrm{ClO}_{4}$, abbreviated as NENP [1], displaying no magnetic long-range order down to very low temperatures. Over the years, other real spin chains with varying anisotropies have been synthesised and measurements of susceptibility, the field-dependent magnetization, ESR and NMR have been performed.

The aim of this report is the verification of some theoretical results and the values of the microscopic parameters selected earlier from different fitting procedures, using reliable numerical simulations based on the unique quantum transfer matrix technique (QTM).

In the presence of the external field, the 1D HAF chains considered here are described by the $S=1$ Hamiltonian

$$
\mathcal{H}=J \sum_{i=1}^{N-1} S_{i} S_{i+1}+D \sum_{i=1}^{N}\left(S_{i}^{z}\right)^{2}-g_{\alpha} \mu_{\mathrm{B}} B \sum_{i=1}^{N} S_{i}^{\alpha},
$$

where $J(>0)$ denotes the antiferromagnetic interaction constant, $D$ stands for the anisotropy parameter, $B$ is the external magnetic field which can be applied along the chain $(\alpha=z)$ or in the perpendicular direction $(\alpha=x)$ and $g_{\alpha}$ is the corresponding gyromagnetic ratio. 
A number of simulation techniques have been applied to 1D IIAF chains. IIerein the variant of the quantum transfer-matrix approach proposed by Delica et al. [2] has been adapted and we follow the notation introduced therein. The advantages of the present transfer matrix technique are diverse: no statistical errors, the real-space decomposition scheme, the reduction of the amount of the degrees of freedom and a collapse of all the states under the trace operation.

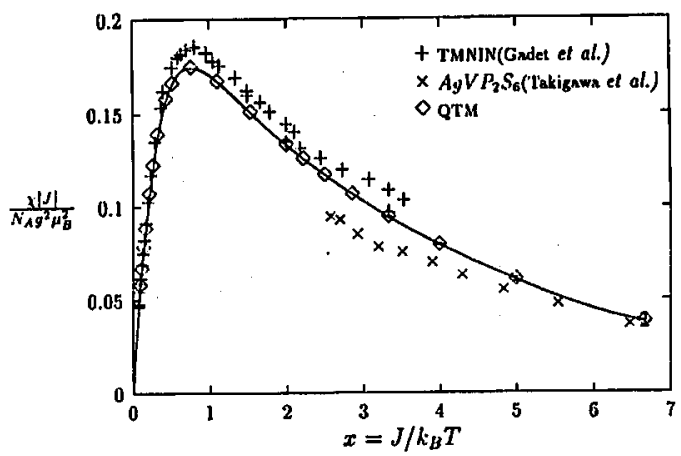

Fig. 1. The isotropic susceptibility in reduced units. The continuous curve fits the numerical QTM data. The remaining symbols represent the experimental data.

The following results might be interesting from the experimental point of view. In Fig. 1 our susceptibility data in the isotropic limit are given and compared with the measurements on TMNIN $\left(\left(\mathrm{CH}_{3}\right)_{4} \mathrm{NNi}\left(\mathrm{NO}_{2}\right)_{3}\right)$ ) [3] and $\mathrm{AgVP}_{2} \mathrm{~S}_{6}$ [4] viewed as isotropic compounds. We have also derived the Chebyshev polynomial fitting of our susceptibility data in the entire temperature region. The corresponding high temperature expansion of Weng (e.g. [5]) is usually referred to in the interpretation of the susceptibility measurements. Our results (Fig. 1) coincide with those of Weng in the high temperature limit and provide a curve applicable down to lower temperatures.

We performed extensive calculations in the interval of parameters appropriate for NENP. From our simulations we have found the best overall fit for the following parameters:

$$
J / k_{\mathrm{B}}=48 \mathrm{~K}, \quad D / k_{\mathrm{B}}=7.8 \mathrm{~K}, \quad g_{\perp}=2.25, \quad g_{\|}=2.20 .
$$

The results for NENP are summarized in Figs. 2 and 3, where we display the susceptibility as well as the low-field magnetization data both calculated here and measured experimentally.

Our simulations have some theoretical consequences, too. Firstly, we report our test of the theoretical findings of Regnault et al. [6] in a boson and fermion approximation. We have revealed discrepancies in the behaviour of both susceptibility and specific heat. Secondly, we consider the strong anisotropy limit, to test the strong coupling approach and a choice of parameters for NENC $\left(\mathrm{Ni}\left(\mathrm{C}_{2} \mathrm{II}_{8} \mathrm{~N}_{2}\right)_{2} \mathrm{Ni}(\mathrm{CN})_{4}\right)$ [7]. In this case our results are consistent with those coming from the analytical approach. 


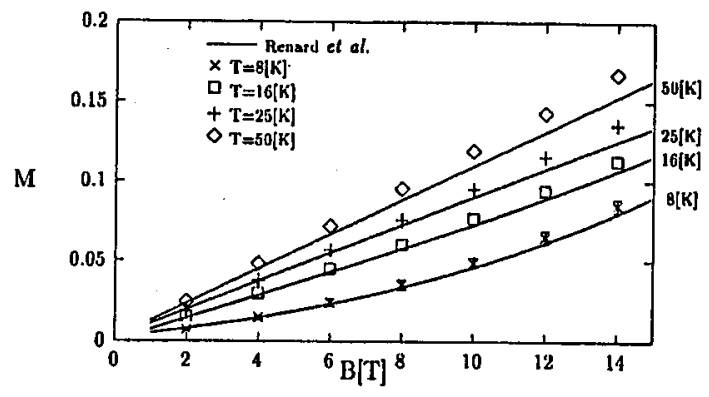

Fig. 2. The low-field magnetization data for NENP. The QTM data are shown by the symbols and experimental data by the continuous line.

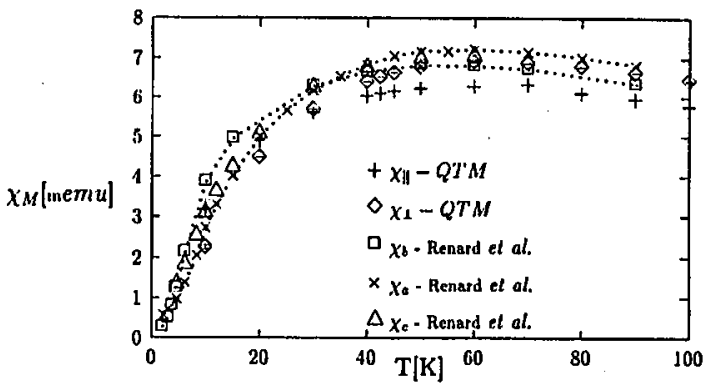

Fig. 3. The molar susceptibility for NENP. The dotted lines are drawn to distinguish the numerical values from the experimental findings.

An account of the simulation results for the ferromagnetic compound $\mathrm{CsNiF}_{3}$ has been published elsewhere [8].

This work was supported in part by the Committee for Scientific Research under the grant No. 2 P302 116 06. The numerical calculations were carried out in the Supercomputing and Networking Center in Poznań.

\section{References}

[1] J.P. Renard, M. Verdagner, L.P. Regnault, W.A.C. Erkelens, J. Rossat-Mignad, J. Ribas, W.G. Stirling, C. Vettier, J. Appl. Phys. 63, 3538 (1988).

[2] T. Delica, K. Kopinga, II. Lesclke, K.K. Mon, Europhys. Lett. 15, 55 (1991).

[3] V. Gadet, M. Verdagner, V. Briois, A. Glcizes, J.P. Renard, P. Beauvillain, C. Chappert, T. Goto, K. Le Dang, P. Veillet, Phys. Rev. B 44, 705 (1991).

[4] M. Takigawa, T. Asano, Y. Ajiro, M. Mekata, Phys. Rev. B 52, R13087 (1995).

[5] J. Darriet, L.P. Regnault, Solid Stale Commun. 86, 409 (1993).

[6] L.P. Regnault, I. Zaliznyak, S. Meshkov, J. Phys., Condens. Matter 5, L677 (1993).

[7] N. Papanicolaou, P.N. Spathis, Phys. Rev, B 52, 16001 (1995).

[8] L.S. Campana, A. Caramico D'Auria, F. Esposito, U. Esposito, G. Kamieniarz, Phys. Rev. B 45, 5035 (1992). 